\title{
Hybridization capture reveals microbial diversity missed using current profiling methods
}

\author{
Cyrielle Gasc and Pierre Peyret ${ }^{*}$
}

\begin{abstract}
Background: Microorganisms comprise the majority of living organisms on our planet. For many years, exploration of the composition of microbial communities has been performed through the PCR-based study of the small subunit rRNA gene due to its high conservation across the domains of life. The application of this method has resulted in the discovery of many unexpected evolutionary lineages. However, amplicon sequencing is subject to numerous biases, with some taxa being missed, and is limited by the read length of second-generation sequencing platforms, which drastically reduces the phylogenetic resolution.

Results: Here, we describe a hybridization capture strategy that allows the enrichment of 165 rRNA genes from metagenomic samples and enables an exhaustive identification and a complete reconstruction of the biomarker. Applying this approach to a microbial mock community and a soil sample, we demonstrated that hybridization capture is able to reveal greater microbial diversity than $16 \mathrm{~S}$ rDNA amplicon sequencing and shotgun sequencing. The reconstruction of full-length $16 \mathrm{~S}$ rRNA genes facilitated the improvement of phylogenetic resolution and the discovery of novel prokaryotic taxa.

Conclusions: Our results demonstrate that hybridization capture can lead to major breakthroughs in our understanding of microbial diversity, overcoming the limitations of conventional $16 \mathrm{~S}$ rRNA gene studies. If applied to a broad range of environmental samples, this innovative approach could reveal the undescribed diversity of the still underexplored microbial communities and could provide a better understanding of ecosystem function.
\end{abstract}

Keywords: Hybridization capture, Microbial community profiling, 16S rRNA gene

\section{Background}

With an estimated total number of $4-6 \times 10^{30}$ cells, prokaryotes are the most diverse and abundant cellular life forms on Earth $[1,2]$. With the advent of PCR and highthroughput next-generation sequencing (NGS) technologies, the small subunit rRNA gene has become the most widely used marker for molecular ecology, providing microbial community diversity information in a cultivationindependent manner [3]. PCR-based studies targeting the $16 \mathrm{~S}$ rRNA gene have led to the discovery of many unexpected evolutionary lineages [4]. However, partial 16S rRNA gene sequences produced from NGS short-read

\footnotetext{
* Correspondence: pierre.peyret@uca.fr

Université Clermont Auvergne, INRA, UMR 454 MEDIS, 28, place Henri Dunant, F-63000 Clermont-Ferrand, France

sequencing platforms often result in incorrect or inaccurate taxonomic assignment of amplicons [5] and do not reflect community diversity [6]. Moreover, amplicon sequencing is subject to numerous biases such as differential amplification efficiencies, preferential amplification of specific targets, and unability of degenerate primer to target all the intended targets [6, 7]. Shotgun reads obtained from metagenomic studies provide a source of sequences that are not subject to these major concerns and give access to longer $16 \mathrm{~S}$ rDNA sequences that enhance phylogenetic assignment [5]. However, because of the short and random nature of metagenomic sequences, most of the informative regions of the 16S rRNA genes might be missed. Indeed, shotgun sequencing of metagenomics samples preferentially provides sequences of dominant microorganisms, thus diminishing the phylogenetic description of microbial 
communities. Several methodological $[8,9]$ or bioinformatic $[10,11]$ strategies have been developed to recover complete or near-complete rRNA genes, but all of them suffer major limitations linked to the difficulties inherent in completely exploring complex microbial diversity.

To overcome the limitations related to microbial communities profiling with conventional molecular approaches, we developed a new hybridization capture method that allows the targeted enrichment of $16 \mathrm{~S}$ rRNA genes from microbial communities. Gene capture approaches by hybridization traditionally use tiling probes to specifically target and enrich specific biomarkers or genomic regions from genomic DNA isolated from model organisms for resequencing experiments in order to identify new genetic variants [12]. Nevertheless, hybridization capture also allows the capture of divergent targets as it is often done for ancient DNA capture or non-reference species capture. The impact of sequence divergence on the efficiency of hybridization capture has already been evaluated. For example, Hedtke et al. [13] used exome capture to enrich DNA across frog species spanning approximately 250 million years of evolutionary divergence (up to approximately $10 \%$ divergence). Thus, hybridization authorizes mismatches between probes and distant targeted sequences not already referred in database. We demonstrated the efficiency of such sequence capture to explore the methanogenic communities present in a lacustrine environment by targeting the methyl coenzyme $\mathrm{M}$ reductase subunit A $(m c r A)$ gene with a set of nonoverlapping probes, which targeted both known sequences and potential undescribed variants of the $m c r A$ gene [14]. Here, we report the first application of a hybridization capture strategy [15] that uses a set of probes targeting all known 16S rRNA gene bacterial and archaeal diversity to enrich the full-length biomarker and to explore the microbial diversity of a metagenomic soil sample contaminated with hexachlorocyclohexane $(\mathrm{HCH})$. To evaluate the efficiency of this strategy, we applied this approach to a microbial mock community and we compared it to 16S rRNA gene amplicon sequencing and shotgun sequencing approaches.

\section{Methods}

\section{Microbial mock community}

An artificial mixture composed of 21 bacterial and 7 archaeal species (representing 6 phyla, 13 classes, 19 orders, 23 families, and 26 genera) whose genome are sequenced was made from genomic DNA extracted from pure cultures of the different species (DSMZ) (Additional file 1: Table S1). Abundances of the different species based on the 16S rDNA copy number per genome and the number of genomes in the mixture was defined so that the final community profile reflects the abundance variability of species in an environmental microbial community (Additional file 1: Table S1).

\section{DNA isolation from soil}

A genomic DNA sample was extracted from a hexachlorocyclohexane $(\mathrm{HCH})$-contaminated soil sample collected from an old chemical factory (Huningue, France) using a PowerSoil DNA Isolation Kit (MoBio).

\section{Shotgun sequencing of the samples}

Two NGS libraries were constructed from the microbial mock DNA mixture and the soil genomic DNA using the Nextera and TruSeq Kits (Illumina), respectively, according to the manufacturer's instructions. Libraries were directly sequenced in two paired-end $(2 \times 250 \mathrm{bp})$ MiSeq runs (Illumina).

\section{$16 \mathrm{~S}$ rRNA gene amplicon sequencing}

An approximately 300-bp fragment from the V4 variable region of the $16 \mathrm{~S}$ rRNA genes was amplified from the microbial mock DNA mixture and the soil genomic DNA by PCR using "universal" primers F515 (GTGCCAGCMGCCGCGGTAA) and R806 (GGACTACHVGGGTWTCTAAT) [16]. Two sequencing libraries were then constructed following the TruSeq DNA library preparation protocol (Illumina), and sequencing of the amplicons was performed on the MiSeq platform $(2 \times 300 \mathrm{bp}$, Illumina $)$.

\section{Hybridization capture targeting the 16S rRNA gene}

For probe design, a 16S rDNA-curated sequence database corresponding to all 16S rRNA gene diversity was constructed from the EMBL [17]. A set of 28- to 50-mer degenerate probes (Additional file 1: Table S2) was designed from these databases using KASpOD [18] and PhylArray [19] software that enable the determination of degenerate explorative capture probes. A minimal probe set composed of 15 probes has been selected so that probes are distributed over the entire length of the gene and can hybridize all the known $16 \mathrm{~S}$ rRNA gene sequences used for probe design and potentially other $16 \mathrm{~S}$ rDNA variants never described in databases. Probe length has been selected so that probes have a medium size and a GC content (Additional file 1: Table S2) that provides a good compromise between sensitivity and specificity and that allows some mismatches between probes and their targets to enrich all the $16 \mathrm{~S}$ gene diversity [20]. Adaptor sequences were added to the ends of the probes to enable their amplification by PCR, resulting in "ATCGCACCAGCGTGT- $\mathrm{N}_{\mathrm{X}}$-CACTGCGGCTCCTCA" sequences, with $\mathrm{N}_{X}$ representing the $16 \mathrm{~S}$ rRNA gene-specific capture probes. Biotinylated RNA capture probes were then synthesized as described by Ribière et al. [21]. In brief, adaptors containing the $\mathrm{T} 7$ promoter were added to the $16 \mathrm{~S}$ rRNA gene-specific capture probes via ligationmediated PCR, and the final biotinylated RNA probes were obtained after in vitro transcription and purification. 
To perform hybridization capture, $2.5 \mu \mathrm{g}$ of salmon sperm DNA (Ambion) and $500 \mathrm{ng}$ of denatured Illumina library constructed from the microbial mock community or the soil sample were mixed, denatured for $5 \mathrm{~min}$ at $95^{\circ}$ $\mathrm{C}$, and incubated for $5 \mathrm{~min}$ at $65^{\circ} \mathrm{C}$ before adding $13 \mu \mathrm{l}$ of prewarmed $\left(65{ }^{\circ} \mathrm{C}\right)$ hybridization buffer (10X SSPE, 10X Denhardt's solution, $10 \mathrm{mM}$ EDTA and 0.2\% SDS) and $500 \mathrm{ng}$ of prewarmed $\left(65^{\circ} \mathrm{C}\right)$ biotinylated RNA probes. After hybridization at $65{ }^{\circ} \mathrm{C}$ for $24 \mathrm{~h}$, the probe/target heteroduplexes were captured using $500 \mathrm{ng}$ of washed streptavidin-coated paramagnetic beads (Dynabeads M-280 Streptavidin, Invitrogen). The beads were collected using a magnetic stand (Ambion) and washed once at room temperature with $500 \mu \mathrm{l} 1 \mathrm{X} \mathrm{SSC/0.1 \%} \mathrm{SDS} \mathrm{and}$ three times at $65{ }^{\circ} \mathrm{C}$ with $500 \mu \mathrm{l}$ prewarmed $0.1 \mathrm{X} \mathrm{SSC/}$ $0.1 \%$ SDS. The captured fragments were eluted with $50 \mu \mathrm{l}$ $0.1 \mathrm{M} \mathrm{NaOH}$. After magnetic bead collection, the DNA supernatant was transferred to a sterile tube containing $70 \mu \mathrm{l}$ of $1 \mathrm{M}$ Tris- $\mathrm{HCl} \mathrm{pH} 7.5$ and PCR-amplified using primers complementary to the library adapters. To increase the enrichment efficiency, a second round of hybridization capture was performed using the first-round capture products. Enriched DNA from the mock community and the soil sample were then sequenced using two Illumina MiSeq $2 \times 250$ bp runs.

\section{Sequencing data processing Microbial mock community}

Reads obtained from the shotgun sequencing, amplicon sequencing, and hybridization capture from the microbial DNA mixture were deposited in the NCBI open access sequence read archive (SRA) under accession numbers SRR5381736, SRR5381734, and SRR5381738, respectively. Reads were scanned for library adaptors and quality-filtered using the PRINSEQ-lite PERL script [22] prior to analysis. After filtering, 1,097,064 pairs of reads were obtained from the shotgun sequencing library, 9,259,211 were obtained from the hybridization capture library, and 8,218,732 pairs of reads were obtained from the amplicon sequencing library. For comparison, the sequence number of each sample was randomly normalized to the same sequencing depth, i.e., 1,097,064 paired-end sequences per sample.

The proportions of $16 \mathrm{~S}$ rRNA gene sequences were estimated in the three datasets using SortMeRNA [23] with the default parameters.

Reads obtained from the three methods were mapped against the 1,922,213 16S rDNA sequences from Silva SSURef 128 database using Bowtie2 (V2.1.0) [24] with endto-end very sensitive mode. The number of reads aligned in each $16 \mathrm{~S}$ rDNA sequence from Silva database and the coverage per sequence position were calculated using SAMtools 1.3 [25]. Genera totaling more than 200 mapped reads were considered as present in the samples, which represents an average coverage of $30 \mathrm{X}$ over the entire length of the $16 \mathrm{~S}$ rDNA. Such coverage enables the complete and unambiguous reconstruction of the biomarker.

\section{Soil sample}

Reads obtained from the shotgun sequencing, amplicon sequencing, and hybridization capture from the soil sample were deposited in the NCBI open access sequence read archive (SRA) under accession numbers SRR3546814, SRR3648004, and SRR3654007, respectively. Reads were scanned for library adaptors and quality-filtered using the PRINSEQ-lite PERL script [22] prior to analysis. After filtering, 19,377,521 pairs of reads were obtained from the shotgun sequencing library, 3,719,256 were obtained from the hybridization capture library, and 529,078 pairs of reads were obtained from the amplicon sequencing library. Sequences of each sample were not randomly normalized to the same sequencing depth. Indeed, the sequencing depth is inversely proportional to the number of $16 \mathrm{~S}$ rRNA gene sequences expected for each sample: a lower sequencing depth is required for amplicon sequencing where approximately all sequences correspond to the 16S rRNA gene, deep sequencing is necessary for shotgun metagenomics where the biomarker is presumed to represent a very small fraction of the reads, and an intermediate depth is suitable for hybridization capture where a portion of the reads are of interest but still represent the majority of the data compared with shotgun sequencing as a consequence of the targeted enrichment.

The proportions of $16 \mathrm{~S}$ rRNA gene sequences were estimated in the three datasets using SortMeRNA [23] with the default parameters. 16S rRNA gene reconstruction and OTU clustering from the three samples were performed using EMIRGE 0.60 [11]. Chimeric sequences were eliminated with Uchime 4.2.40 [26] using the ChimeraSlayer "Gold" database using a 0.28 cutoff score. Taxonomic classification of the sequences was then made with RDP Classifier [27] using the Silva [28] database 119 release with a confidence cutoff set at 0.5 . We did not correct the microbial abundance considering the $16 \mathrm{~S}$ copy number variation among taxa because we favored the overall comparison of the three molecular methods rather than the precise description of microbial communities in our soil sample.

Classification of the unassigned 16S rDNA sequences was performed as described by Flandrois et al. [29]. In brief, BLASTN was run on the $16 \mathrm{~S}$ rDNA sequences against the SSU rDNA stringent database containing 234,263 bacterial and archaeal sequences from GenBank with the expectation value set to 0.1 . The 50 sequences with the highest similarity scores were extracted, and multiple alignments were generated against the query sequence using MAFFT [30]. FastTree [31] was then used to reconstruct the tree by approximate maximum likelihood using the general time reversible (GTR) model. Branch support was calculated 
with the Shimodaira-Hasegawa $(\mathrm{SH})$ test. The 12 novel 16S rDNA sequences described in the present work were deposited in the Genbank database under accession numbers KX363569 to KX363580.

The distance matrixes between the unassigned 16S rDNA sequences and the closest representative sequences identified through phylogenetic placement were performed with Clustal Omega [32]. The thresholds were set at 80, 85, and $90 \%$ identity to define the taxonomic phylum, class, and order levels, respectively.

\section{Results and discussion}

We developed a hybridization capture strategy that uses a set of 15 degenerate explorative probes targeting $16 \mathrm{~S}$ rRNA gene bacterial and archaeal diversity to enrich the full-length biomarker and to explore the microbial diversity of a metagenomic samples (Additional file 1: Figure S1). Enrichment relies on the ability of probes designed on highly conserved regions along the 16S rRNA reference genes to specifically capture DNA from a broad range of species. In the present study, we validated our method on a microbial mock community composed of 28 species and compared it with shotgun sequencing and 16S rRNA gene amplicon sequencing with the widely used F515-R806 primer pair [16]. To demonstrate the efficiency of the method on a real microbial community, we then applied this approach to characterize the microbial structure of a metagenomic soil sample.

\section{Validation on a microbial mock community}

We first applied our hybridization capture method, amplicon sequencing, and shotgun sequencing to a synthetic metagenome composed of DNA extracted from 21 bacterial and 7 archaeal species belonging to 26 phylogenetically distant genera (Additional file 1: Table S1). Relative abundances of the different species have been selected so that the final community profile reflects the species abundance variability in an environmental microbial community, with abundant microorganisms (e.g., Clostridium acetobutylicum representing $32.63 \%$ of the community) and microorganisms belonging to the rare biosphere (e.g., Methanobrevibacter smithii and Metahnococcus aeolicus, each accounting for $0.00006 \%$ of the community).

Firstly, we calculated the proportion of reads reflecting $16 \mathrm{~S}$ rRNA genes in the three datasets and demonstrated that hybridization capture leads to a significant enrichment of the 16S rRNA gene. Indeed, $97.30 \%$ of reads correspond to the $16 \mathrm{~S}$ rRNA gene for hybridization capture compared with shotgun sequencing in which only $0.41 \%$ of reads carry the phylogenetic biomarker. Thus, hybridization capture enables an optimal enrichment comparable to that obtained with amplicon sequencing (99.52\%).

We then characterized the community structures obtained using the three methods through mapping of the reads against the $16 \mathrm{~S}$ rDNA sequences of the Silva database. In this way, we showed that with hybridization capture, 24 of the 26 genera are detected and that the $16 \mathrm{~S}$ reference sequences of these genera are covered over their entire length with a minimum coverage of 180X (Fig. 1). The 24 genera detected, ranging from 32.63\% (Clostridium) to $0.00059 \%$ (Sphingobium) of the community, demonstrate the ability of the method to detect rare microorganisms. Thus, only the two less abundant species, Methanobrevibacter smithii and Methanococcus aeolicus $(0.00006 \%$ each), are missed by hybridization capture with this sequencing depth $(1,097,064$ pairs of reads). However, when mapping the hybridization capture reads prior to their normalization $(9,259,211$ pairs of reads), Methanobrevibacter smithii $(0.00006 \%)$ is also detected in the sample bringing to 25 out of 26 the number of genera detected and decreasing the detection limit to $0.00006 \%$. With shotgun sequencing, only 10 dominant genera are identified in the dataset because of the difficulty of detecting the less abundant species in metagenomic samples even at high sequencing depths (Fig. 1). Finally, with amplicon sequencing, 23 out of the 26 genera are detected (32.63 to $0.00059 \%$ of the community), but contrary to hybridization capture and shotgun sequencing, this method also detects 49 other genera (ranging from 1.50 to $0.01 \%$ of the community) that are not part of the mock community (Fig. 1). This overestimation of diversity can be explained by the inaccurate assignment of the short fragments amplified by the primer pair, leading to a biased description of communities. Finally, by covering the complete 16S rDNA sequences, reads obtained through hybridization capture allow an accurate assignment of sequences up to the species and most of the time up to the strain level. In this way, hybridization capture clearly distinguishes the different species in the mock community contrary to amplicon sequencing that overestimates the diversity at low-resolution taxonomic levels. As a consequence, at the genus level but also at all the other taxonomic ranks, the description of community differs between the three methods (Fig. 1, Additional file 1: Figure S2). Indeed, in addition to detecting nearly all of the species present in the sample, hybridization capture yields abundances for the different species close to that expected (Additional file 1: Table S1). Even if slight variations of abundance tending to overestimate the rare species appear between the theoretical and hybridization capture profiles $(1.63 \pm 2.13 \%$ when abundance is $\geq 1 \% ; 0.14 \pm 0.18 \%$ when abundance is $\leq 1 \%$ ), the relative abundance of species in the community is well estimated.

Thus, the use of hybridization capture on this microbial mock community demonstrates that the method gives a vision very close to the expected community structure with a high sensitivity, contrary to shotgun sequencing that gives a description restricted to the 


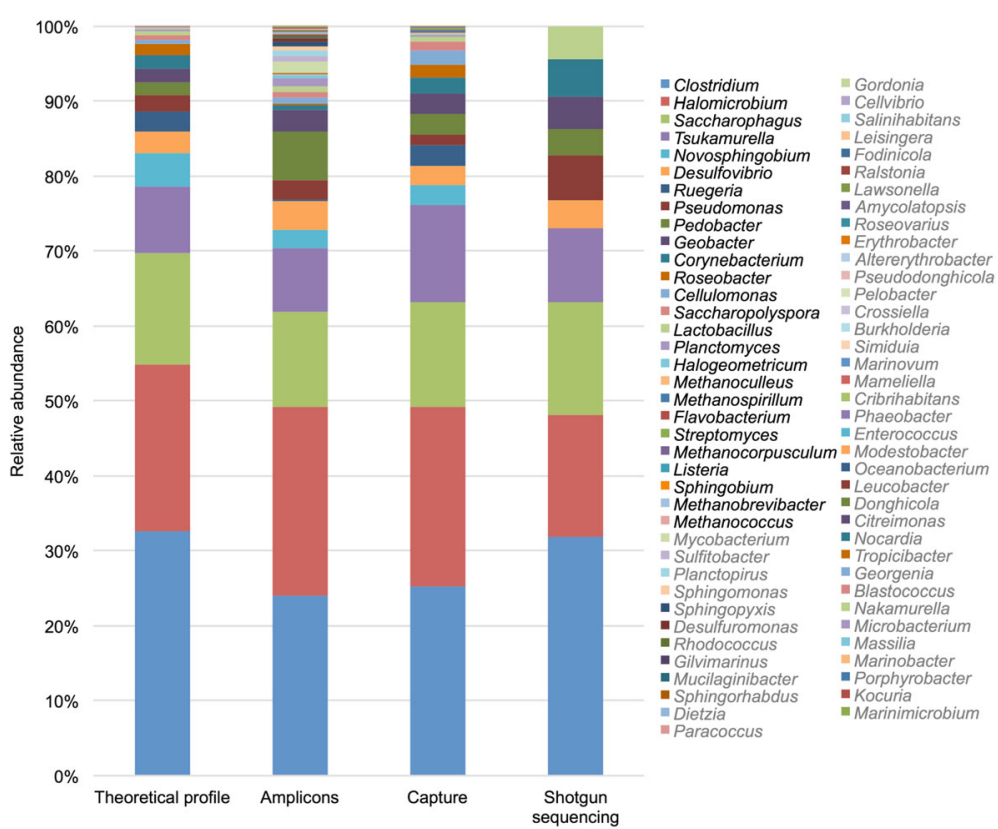

Fig. 1 Microbial mock community profiles at the genus level for amplicon sequencing, hybridization capture, and shotgun sequencing. The genera identified through amplicon sequencing that are not part of the microbial mock community are indicated in gray. The abundance profiles at other taxonomic levels for the three datasets are available in Additional file 1: Figure S2

dominant species and to amplicon sequencing that does not allow an accurate assignment of reads and gives a biased vision of diversity. Further hybridization capture experiments could be carried out on the same microbial mock community to assess the reproducibility of the method. However, the high reproducibility of hybridization capture experiments performed on other biological models such as human exome [33-35] has already been demonstrated.

\section{Soil microbial community profiling}

We then applied our hybridization capture method to a soil sample, considered as the most complex metagenomic sample, to demonstrate its efficiency on real microbial communities. As previously, we first estimated the proportion of reads reflecting 16S rRNA genes sequenced using the different approaches and demonstrated that hybridization capture also leads to a significant enrichment of the targeted biomarker in this complex sample. Indeed, with hybridization capture, the $16 \mathrm{~S}$ rRNA gene represents $58.74 \%$ of the reads compared with shotgun sequencing, in which only $0.09 \%$ of sequences carry the biomarker. Even if significant and sufficient for the description of communities, this enrichment is probably less important than the enrichment obtained with the mock community because of the bigger complexity of this soil sample.
We then conducted operational taxonomic unit (OTU) reconstruction for the 16S rRNA genes from the three datasets using the dedicated EMIRGE software [11] and classified them with the Silva [28] database after chimera detection. At all taxonomic ranks, from domain to species, the community structures differed between the three methods (Fig. 2, Additional file 1: Figure S3). Indeed, 354 OTUs representing species were identified through capture compared with 13 OTUs through shotgun sequencing and 115 OTUs through amplicon sequencing. The lack of OTUs identified by shotgun sequencing is likely attributable to the complexity of the soil ecosystem and the difficulty of obtaining exhaustive $16 \mathrm{~S}$ rRNA gene sequences even at very high sequencing depths. Similarly, amplicon sequencing is limited by the inability of "universal" primer pairs to target all the diversity of the 16S rRNA genes and by the possible inefficient amplification of complex metagenomic samples (Fig. 2, Table 1).

We observed that with hybridization capture, bacteria (99.63\% of the $16 \mathrm{~S}$ rDNA reads) and archaea $(0.37 \%$ of the $16 \mathrm{~S}$ rDNA reads) were simultaneously revealed, in contrast to the other approaches (Additional file 1: Figure S3a), even though the primer pair used for $16 \mathrm{~S}$ amplicon sequencing also targets archaea [36] as demonstrated when studying the mock community. Archaea are relatively rare in soil, with Thaumarchaeota being the dominant archaeal group [37], 


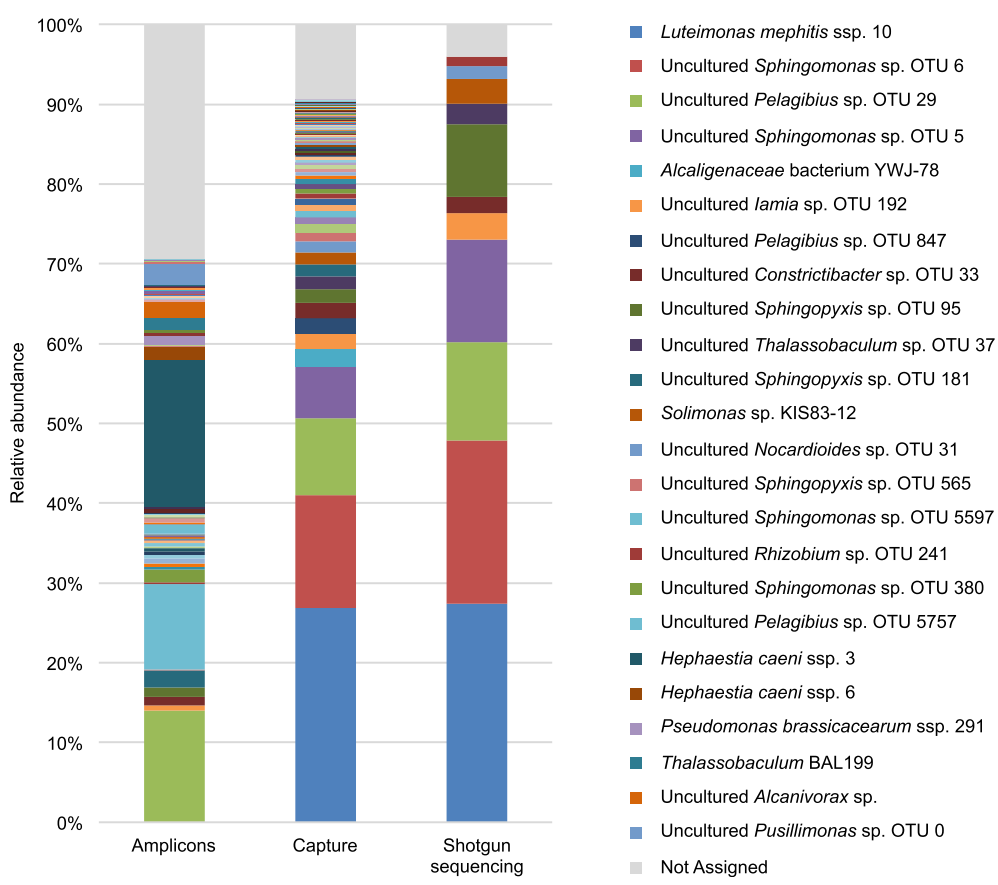

Fig. 2 Soil composition profiles at the species level for amplicon sequencing, hybridization capture, and shotgun sequencing. Only the dominant bacterial species (relative abundance $>1 \%$ ) are indicated in the legend. The abundance profiles at other taxonomic levels for the three datasets are available in Additional file 1: Figure S3

as we observed by gene capture, revealing the sensitivity of this approach. Despite the differences in the structures of the microbial communities identified between the three methods, the same environmental taxa dominate the three datasets at low-resolution classification (Additional file 1: Figure S3). Proteobacteria, known to be abundant in soil [37] and to comprise many $\mathrm{HCH}$-degrading species, form the dominant phylum in the three samples, representing $92.09,89.53$, and $93.44 \%$ of the reads by amplicon sequencing, hybridization capture, and shotgun sequencing, respectively. The Proteobacteria were primarily represented by Alphaproteobacteria (65.97, 53.24 , and $62.94 \%$ of the reads, respectively) and
Gammaproteobacteria $(20.95,32.35$, and $30.50 \%$ of the reads, respectively) (Additional file 1: Figure S3b, c). In regard to the family level, Sphingomonadaceae (30.91 to $42.45 \%$ of the reads), Xanthomonadaceae (13.56 to $29.78 \%$ of the reads), and Rhodospirillaceae (14.93 to $18.40 \%$ of the reads) dominate the three datasets (Additional file 1: Figure S3e). However, at the genus level, 16S rRNA gene amplicon sequencing almost failed to detect Luteimonas $(0.20 \%$ of the reads in contrast to 27.35 and $27.99 \%$ of the reads through shotgun sequencing and hybridization capture, respectively), one of the three dominant taxa of Sphingomonas (24.14 to $33.36 \%$ of the reads), and Pelagibius (12.21 to $15.47 \%$ of the reads) identified

Table 1 Classification of 165 rDNA sequences into different taxonomic levels. Numbers in parentheses indicate the number of corresponding operational taxonomic units

\begin{tabular}{|c|c|c|c|c|c|c|}
\hline \multirow[b]{2}{*}{ Taxonomic level } & \multicolumn{2}{|c|}{ Hybridization capture } & \multicolumn{2}{|l|}{ Amplicons } & \multicolumn{2}{|c|}{ Shotgun sequencing } \\
\hline & No. of taxa & $\%$ unassigned sequences & No. of taxa & $\%$ unassigned sequences & No. of taxa & $\%$ unassigned sequences \\
\hline Domain & $2(354)$ & $0(0)$ & $1(115)$ & $0(0)$ & $1(13)$ & $0(0)$ \\
\hline Phylum & $18(353)$ & $0.007(1)$ & $8(115)$ & $0(0)$ & $3(13)$ & $0(0)$ \\
\hline Class & $42(352)$ & $0.014(2)$ & $16(115)$ & $0(0)$ & $5(13)$ & $0(0)$ \\
\hline Order & 73 (338) & $0.284(16)$ & $29(114)$ & $0.295(1)$ & $8(13)$ & $0(0)$ \\
\hline Family & $117(307)$ & $1.260(47)$ & $35(112)$ & $0.543(3)$ & $9(13)$ & $0(0)$ \\
\hline Genus & $143(263)$ & $4.600(91)$ & $48(100)$ & $11.797(15)$ & $12(13)$ & $0(0)$ \\
\hline Species & 190 (190) & 9.419 (164) & 73 (73) & $29.464(42)$ & $11(11)$ & $4.091(2)$ \\
\hline
\end{tabular}


through hybridization capture and shotgun sequencing (Additional file 1: Figure S3f), thus generating a different profile of the microbial community at the species level (Fig. 2). Indeed, the dominant species identified through hybridization capture and shotgun sequencing, Luteimonas mephitis ssp. 10 (26.81 and $27.26 \%$ of the reads, respectively) and uncultured Sphingomonas sp. OTU 6 (14.21 and 20.52\% of the reads, respectively), were missed by amplicon sequencing, which instead includes among its dominant species Hephaestia caeni ssp. 3 (18.39\% of the reads), a species absent in the two other sequencing datasets. Consequently, whereas all 11 species identified through shotgun sequencing correspond to the dominant species identified using hybridization capture, only 30 of the 73 species identified with amplicon sequencing are shared with those obtained using hybridization capture (Table 1). This difference is most likely due to the inaccurate classification of short rDNA regions from amplicons at such a precise taxonomic level. These results highlight that hybridization capture is able to reveal diversity that is missed using the other two methods.

Another major difference that appears between the three approaches is the percentage of unassigned $16 \mathrm{~S}$ rDNA sequences at the level of species: 4.09, 9.42, and 29.46\%, respectively, for shotgun sequencing, hybridization capture, and PCR sequencing (Fig. 2, Table 1). For PCR sequencing, because of the short amplicon size (approximately $300 \mathrm{bp}$ ), accurate phylogenetic assignment of sequences is possible down to the taxonomic order or family but is unachievable at the genus level for most of sequences. By contrast, hybridization capture and shotgun sequencing allow for the reconstruction of complete or almost complete $(1385 \pm 121$ and $1239 \pm 253 \mathrm{bp}$, respectively) $16 \mathrm{~S}$ rRNA genes, thereby facilitating accurate taxonomic classification. However, even with a full-length $16 \mathrm{~S}$ rRNA gene, OTUs remain unassigned with hybridization capture, demonstrating its capability of revealing novel $16 \mathrm{~S}$ rDNA sequences not described in current databases.

\section{Discovery of new taxa}

To further demonstrate the capability of hybridization capture to reveal novel sequences, we analyzed the unassigned 16S rDNA sequences at low-resolution taxonomic levels through the placement of the sequences into phylogenetic trees. Analysis of a full-length $16 \mathrm{~S}$ rDNA sequence not assigned at the level of phylum revealed that the sequence forms a discrete clade (Fig. 3, Additional file 1: Figure S4a) close to the Nitrospirae. Alignment of the $16 \mathrm{~S}$ rRNA gene with the closest sequences (Additional file 1: Figure S4b) based on this phylogeny and an exhaustive environmental phylogeny [38] revealed that the unidentified $16 \mathrm{~S}$ rDNA sequence was less than $80 \%$ identical to the other sequences, suggesting that this $16 \mathrm{~S}$ rRNA gene belongs to a new candidate phylum. Similarity searches in $\mathrm{nr}$ database from NCBI revealed two identical environmental sequences (JN607053.1, DQ499315.1) identified as "uncultured bacterium" isolated from microorganisms from two different cave soils. We also reanalyzed the unassigned $16 \mathrm{~S}$ rDNA sequences for hierarchical class and order and determined their probable classifications to new taxa. Indeed, we assigned one sequence attributed to the Gemmatimonadetes phylum to a new class (Additional file 1:

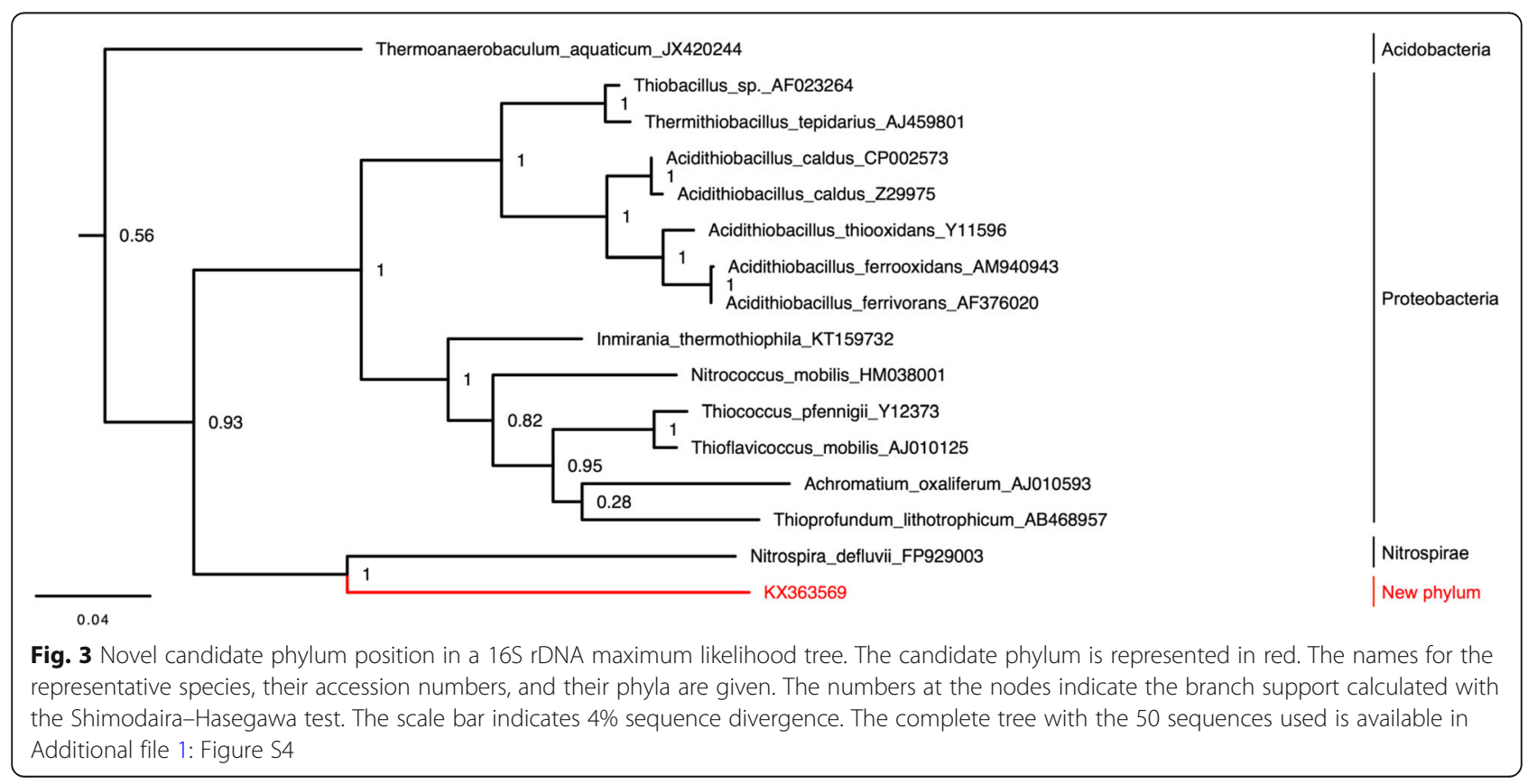


Figure S5), another sequence to a new class belonging to the Chloroflexi (Additional file 1: Figure S6), and nine new sequences to two Saccharibacteria (Candidate Division TM7) orders (Additional file 1: Figure S7).

\section{Conclusions}

Here, we demonstrate that hybridization capture targeting the rRNA gene represents a promising approach for microbial community studies. Through the significant enrichment of the 16S rRNA gene (up to $97.30 \%$ of reads), hybridization capture gives an accurate description of communities compared to other conventional molecular methods. Indeed, as evidenced by our results, 16S rRNA gene amplicon sequencing and shotgun sequencing missed taxa, regardless of their abundance in the sample, leading to different community structure descriptions at the genus and species levels. Hybridization capture correlates with conventional approaches at lowresolution taxonomic classification but reveals a broader microbial diversity with its capacity to target even the least abundant species through efficient 16S rRNA gene enrichment. Indeed, based on our experiments, hybridization capture is able to reveal microorganisms that only represent $0.00006 \%$ of the community. Therefore, because of its sensitivity and the use of explorative probes, this hybridization capture method enables the identification of $16 \mathrm{~S}$ rRNA genes belonging to new taxa, allowing the discovery of novelty from the phylum to the species level. Significantly increasing the sequencing depths of shotgun and amplicon sequencing could reveal greater 16S rRNA gene diversity. Nevertheless, for the latter, because of PCR biases, all taxa will never be amplified commensurately, and artificial diversity can be created. Moreover, due to the small sizes of the sequences generated, accurate phylogenetic assignment of reads is infeasible at high-resolution taxonomic levels. By contrast, hybridization capture allows for the reconstruction of complete 16S rRNA gene sequences that are needed to accurately confer phylogenetic associations [39, 40]. Hybridization capture targeting rRNA genes could consequently represent an innovative strategy to describe microbial community structure.

\section{Additional file}

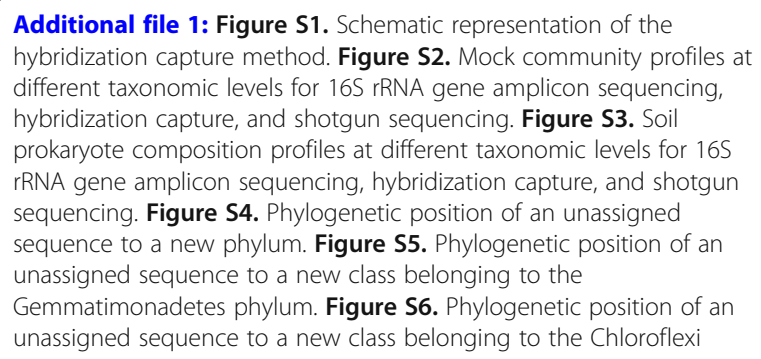

phylum. Figure S7. Phylogenetic position of new unassigned sequences to the Saccharibacteria phylum. Table S1. Microbial mock community used for hybridization capture validation and $16 \mathrm{~S}$ rDNA relative abundances observed using the three methods (amplicons, capture, and shotgun sequencing). Table S2. Set of probes targeting the 165 rRNA gene used for hybridization capture. (DOCX $4852 \mathrm{~kb}$ )

\section{Acknowledgements}

We would like to thank N. Parisot and F. Jaziri for the probe design and J-Y. Richard (SITA Remediation, Suez) for providing the soil sample.

\section{Funding}

This work was supported by the French "Direction Générale de l'Armement" (DGA), the Investissements d'Avenir AMI 2011 VALTEX program, the Auvergne Regional Council, and the European Regional Development Fund (FEDER).

\section{Availability of data and materials}

Reads obtained from the shotgun sequencing, amplicon sequencing, and hybridization capture from the microbial mock community were deposited in the NCBI open access sequence read archive (SRA) under accession numbers SRR5381736, SRR5381734, and SRR5381738, respectively.

Reads obtained from the shotgun sequencing, amplicon sequencing, and hybridization capture from the soil sample were deposited in the NCBI open access SRA under accession numbers SRR3546814, SRR3648004, and SRR3654007, respectively.

The 12 novel $16 \mathrm{~S}$ rDNA sequences described in the present work were deposited in the Genbank database under accession numbers KX363569 to KX363580.

\section{Authors' contributions}

CG and PP designed the experiments, analyzed the data, and wrote the manuscript. CG performed the experiments. Both authors read and approved the final manuscript.

Ethics approval and consent to participate Not applicable

Consent for publication

Not applicable

\section{Competing interests}

The authors declare that they have no competing interests.

\section{Publisher's Note}

Springer Nature remains neutral with regard to jurisdictional claims in published maps and institutional affiliations.

Received: 31 March 2017 Accepted: 13 March 2018

Published online: 27 March 2018

\section{References}

1. Kallmeyer J, Pockalny R, Adhikari RR, Smith DC, D'Hondt S. Global distribution of microbial abundance and biomass in subseafloor sediment. Proc Natl Acad Sci U S A. 2012;109:16213-6.

2. Whitman WB, Coleman DC, Wiebe WJ. Prokaryotes: the unseen majority Proc Natl Acad Sci U S A. 1998:95:6578-83.

3. Woese CR, Kandler O, Wheelis ML. Towards a natural system of organisms: proposal for the domains Archaea, Bacteria, and Eucarya. Proc Natl Acad Sci U S A. 1990;87:4576-9.

4. Sogin ML, Morrison HG, Huber JA, Mark Welch D, Huse SM, Neal PR, Arrieta JM, Herndl GJ. Microbial diversity in the deep sea and the underexplored "rare biosphere". Proc Natl Acad Sci U S A. 2006;103:12115-20.

5. Yarza P, Yilmaz P, Pruesse E, Glockner FO, Ludwig W, Schleifer KH, Whitman WB, Euzeby J, Amann R, Rossello-Mora R. Uniting the classification of cultured and uncultured bacteria and archaea using 165 rRNA gene sequences. Nat Rev Microbiol. 2014;12:635-45.

6. Hamady M, Knight R. Microbial community profiling for human microbiome projects: tools, techniques, and challenges. Genome Res. 2009;19:1141-52. 
7. Green SJ, Venkatramanan R, Naqib A. Deconstructing the polymerase chain reaction: understanding and correcting bias associated with primer degeneracies and primer-template mismatches. PLoS One. 2015;10:e0128122.

8. Zhang Y, Ji P, Wang J, Zhao F. RiboFR-Seq: a novel approach to linking 165 rRNA amplicon profiles to metagenomes. Nucleic Acids Res. 2016;44:e99.

9. Singer E, Bushnell B, Coleman-Derr D, Bowman B, Bowers RM, Levy A, Gies EA, Cheng JF, Copeland A, Klenk HP, et al. High-resolution phylogenetic microbial community profiling. Isme J. 2016;10:2020-32.

10. Yuan C, Lei J, Cole J, Sun Y. Reconstructing $16 \mathrm{~S}$ rRNA genes in metagenomic data. Bioinformatics. 2015;31:i35-43.

11. Miller CS, Baker BJ, Thomas BC, Singer SW, Banfield JF. EMIRGE: reconstruction of full-length ribosomal genes from microbial community short read sequencing data. Genome Biol. 2011;12:R44.

12. Turner EH, Ng SB, Nickerson DA, Shendure J. Methods for genomic partitioning. Annu Rev Genomics Hum Genet. 2009;10:263-84.

13. Hedtke SM, Morgan MJ, Cannatella DC, Hillis DM. Targeted enrichment: maximizing orthologous gene comparisons across deep evolutionary time. PLoS One. 2013;8:e67908

14. Denonfoux J, Parisot N, Dugat-Bony E, Biderre-Petit C, Boucher D, Morgavi DP, Le Paslier D, Peyretaillade E, Peyret P. Gene capture coupled to highthroughput sequencing as a strategy for targeted metagenome exploration. DNA Res. 2013;20:185-96.

15. Gasc C, Peyretaillade E, Peyret P. Sequence capture by hybridization to explore modern and ancient genomic diversity in model and nonmodel organisms. Nucleic Acids Res. 2016;44:4504-18.

16. Caporaso JG, Lauber CL, Walters WA, Berg-Lyons D, Lozupone CA, Turnbaugh PJ, Fierer N, Knight R. Global patterns of 165 rRNA diversity at a depth of millions of sequences per sample. Proc Natl Acad Sci U S A. 2011;108(Suppl 1):4516-22.

17. Jaziri F, Parisot N, Abid A, Denonfoux J, Ribiere C, Gasc C, Boucher D, Brugere JF, Mahul A, Hill DR, et al. PhylOPDb: a 16S rRNA oligonucleotide probe database for prokaryotic identification. Database. 2014;2014:bau036.

18. Parisot N, Denonfoux J, Dugat-Bony E, Peyret P, Peyretaillade E. KASpOD-a web service for highly specific and explorative oligonucleotide design. Bioinformatics. 2012;28:3161-2.

19. Militon C, Rimour S, Missaoui M, Biderre C, Barra V, Hill D, Mone A, Gagne G, Meier $\mathrm{H}$, Peyretaillade E, Peyret P. PhylArray: phylogenetic probe design algorithm for microarray. Bioinformatics. 2007;23:2550-7.

20. Dugat-Bony E, Peyretaillade E, Parisot N, Biderre-Petit C, Jaziri F, Hill D, Rimour $S$, Peyret $P$. Detecting unknown sequences with DNA microarrays: explorative probe design strategies. Environ Microbiol. 2012;14:356-71.

21. Ribiere C, Beugnot R, Parisot N, Gasc C, Defois C, Denonfoux J, Boucher D,

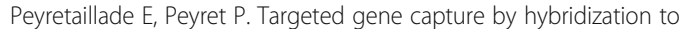
illuminate ecosystem functioning. Methods Mol Biol. 2016;1399:167-82.

22. Schmieder R, Edwards R. Quality control and preprocessing of metagenomic datasets. Bioinformatics. 2011;27:863-4.

23. Kopylova E, Noe L, Touzet H. SortMeRNA: fast and accurate filtering of ribosomal RNAs in metatranscriptomic data. Bioinformatics. 2012;28:3211-7.

24. Langmead B, Salzberg SL. Fast gapped-read alignment with Bowtie 2. Nat Methods. 2012;9:357-9.

25. Li H, Handsaker B, Wysoker A, Fennell T, Ruan J, Homer N, Marth G, Abecasis G, Durbin R. The sequence alignment/map format and SAMtools. Bioinformatics. 2009;25:2078-9.

26. Edgar RC, Haas BJ, Clemente JC, Quince C, Knight R. UCHIME improves sensitivity and speed of chimera detection. Bioinformatics. 2011;27:2194-200.

27. Wang Q, Garrity GM, Tiedje JM, Cole JR. Naive Bayesian classifier for rapid assignment of rRNA sequences into the new bacterial taxonomy. Appl Environ Microbiol. 2007;73:5261-7.

28. Quast C, Pruesse E, Yilmaz P, Gerken J, Schweer T, Yarza P, Peplies J, Glockner FO. The SILVA ribosomal RNA gene database project: improved data processing and web-based tools. Nucleic Acids Res. 2013;41:D590-6.

29. Flandrois JP, Perriere G, Gouy M. leBIBIQBPP: a set of databases and a webtool for automatic phylogenetic analysis of prokaryotic sequences. BMC Bioinformatics. 2015;16:251.

30. Katoh K, Misawa K, Kuma K, Miyata T. MAFFT: a novel method for rapid multiple sequence alignment based on fast Fourier transform. Nucleic Acids Res. 2002;30:3059-66.

31. Price MN, Dehal PS, Arkin AP. FastTree: computing large minimum evolution trees with profiles instead of a distance matrix. Mol Biol Evol. 2009:26:1641-50.

32. Sievers F, Higgins DG. Clustal omega. Curr Protoc Bioinformatics. 2014;48:3-13. 11-16
33. Chilamakuri CS, Lorenz S, Madoui MA, Vodak D, Sun J, Hovig E, Myklebost O, Meza-Zepeda LA. Performance comparison of four exome capture systems for deep sequencing. BMC Genomics. 2014;15:449.

34. Gnirke A, Melnikov A, Maguire J, Rogov P, LeProust EM, Brockman W, Fennell T, Giannoukos G, Fisher S, Russ C, et al. Solution hybrid selection with ultra-long oligonucleotides for massively parallel targeted sequencing. Nat Biotechnol. 2009:27:182-9.

35. Mamanova L, Coffey AJ, Scott CE, Kozarewa I, Turner EH, Kumar A, Howard E, Shendure J, Turner DJ. Target-enrichment strategies for next-generation sequencing. Nat Methods. 2010;7:111-8

36. Walters W, Hyde ER, Berg-Lyons D, Ackermann G, Humphrey G, Parada A, Gilbert JA, Jansson JK, Caporaso JG, Fuhrman JA, et al. Improved bacterial $16 \mathrm{~S}$ rRNA gene (V4 and V4-5) and fungal internal transcribed spacer marker gene primers for microbial community surveys. mSystems. 2015;1(1): e00009-15. https://doi.org/10.1128/mSystems.00009-15.

37. Fierer N, Leff JW, Adams BJ, Nielsen UN, Bates ST, Lauber CL, Owens S, Gilbert JA, Wall DH, Caporaso JG. Cross-biome metagenomic analyses of soil microbial communities and their functional attributes. Proc Natl Acad Sci U S A. 2012;109:21390-5.

38. Hug LA, Baker BJ, Anantharaman K, Brown CT, Probst AJ, Castelle CJ, Butterfield CN, Hernsdorf AW, Amano Y, Ise K, et al. A new view of the tree of life. Nature Microbiology. 2016;1:16048.

39. Liu Z, Lozupone C, Hamady M, Bushman FD, Knight R. Short pyrosequencing reads suffice for accurate microbial community analysis. Nucleic Acids Res. 2007;35:e120.

40. Soergel DA, Dey N, Knight R, Brenner SE. Selection of primers for optimal taxonomic classification of environmental 16S rRNA gene sequences. Isme J. 2012;6:1440-4

\section{Submit your next manuscript to BioMed Central and we will help you at every step:}

- We accept pre-submission inquiries

- Our selector tool helps you to find the most relevant journal

- We provide round the clock customer support

- Convenient online submission

- Thorough peer review

- Inclusion in PubMed and all major indexing services

- Maximum visibility for your research

Submit your manuscript at www.biomedcentral.com/submit

) Biomed Central 